\title{
Estructura factorial y consistencia interna de una versión española del Purpose-In-Life Test*
}

\author{
Factorial Structure and Internal Consistency of a \\ Spanish Version of the Purpose-In-Life Test
}

Recibido: enero 30 de 2012 | Revisado: julio 12 de 2012 | Aceptado: octubre 4 de 2012

\author{
JOAQUÍN GARCÍA-ALANDETE ** \\ Eva Rosa MARTÍNEZ *** \\ PILAR SEllÉS NOHALES ***** \\ Universidad Católica de Valencia, España
}

doi:10.11144/Javeriana.UPSY12-2.efci

Para citar este artículo: García-Alandete, J., Rosa, E. \& Sellés, P. (2013). Estructura factorial y consistencia interna de una versión española del PurposeIn-Life Test. Universitas Psychologica, 12(2), 517-530.

Investigación realizada con el apoyo de Vicerrectorado de Investigación y Calidad de la Universidad Católica de Valencia.

** Profesor en el Departamento de Metodología, Psicología Básica y Psicología Social de la Universidad Católica de Valencia, imparte Historia de la Psicología y Psicología Evolutiva. Profesor de Psicología General en la Facultad de Teología de Valencia (Sección Dominicos). E-mail: ximo. garcia@ucv.es

**** Universidad Católica de Valencia, España. Profesora de Estadística y Psicometría. Departamento de Metodología, Psicología Básica y Psicología Social. Facultad de Psicología y Ciencias de la Salud. Email: eva.rosa@ucv.es

${ }^{* * * * *}$ Universidad Católica de Valencia, España. Profesora de Teorías de la Educación. Departamento de Ciencias de la Ocupación, Psicología Evolutiva y de la Educación. Facultad de Psicología y Ciencias de la Salud.E-mail: pilar.selles@ucv.es

\section{RES UMEN}

El objetivo de este trabajo fue examinar la estructura factorial y la consistencia interna de la versión española del Purpose-In-Life Test, instrumento que mide el logro de sentido de la vida desde los supuestos de la logoterapia. En el estudio participaron 457 universitarios españoles (320 mujeres, 137 hombres) de 18 a 55 años de edad, $M=21.80, D E=4.56$. Se realizaron análisis descriptivos y correlaciones entre los ítems y el total de la escala inicial, un análisis factorial exploratorio, la estimación de la consistencia interna de los factores y de la escala obtenida, el análisis factorial confirmatorio de la misma, la prueba $t$ para comparación de medias entre mujeres y hombres y la prueba de Kruskal-Wallis para el efecto de la edad. Los resultados mostraron una estructura de dos factores correlacionados con aceptable consistencia interna de la escala y de los factores, diferencias significativas entre mujeres y hombres en la puntuación total y uno de los factores, y no significativas en función de la edad. El análisis factorial confirmatorio muestra un adecuado ajuste, apoyando el modelo propuesto.

Palabras clave autores

Purpose-In-Life Test, logoterapia, análisis factorial exploratorio, consistencia interna, análisis factorial confirmatorio.

Palabras clave descriptores

Confiabilidad, validez, psicometría, Investigación cuantitativa.

\footnotetext{
A B S T R A C T

The main objective of this study was to examine the factorial structure and the internal consistency of a Spanish version of Purpose-In-Life Test, which measures the achievement of meaning in life from logotherapy assumptions. Participated 457 Spanish undergraduates (320 females, 137 males) ages 18 to $55 \mathrm{M}=21.8, \mathrm{SD}=4.56$. Estatistical analysis included the descriptive statistics and the correlations of initial scale, an exploratory factor analysis, the internal consistency of the scale and the factors resulting from the factor analysis, a confirmatory factor analysis of resulting scale, and the analysis of the effect due to the gender and the age. Results of exploratory factor analysis showed two correlated factors with acceptable internal consistency, significant differences between women and men in the total score and one of the factors, but not significant depending on the age. Confirmatory factor analysis showed a proper adjustment, supporting the proposed model. Key words authors

Purpose-In-Life, Logotherapy, Exploratory Factor Analysis, Internal Consistency, Confirmatory Factor Analysis.

Key words plus

Reliability, Validity, Psychometry, Quantitative Psychology.
} 
Una cuestión fundamental en cualquier ámbito científico es disponer de instrumentos de medición fiables, válidos y con una estructura precisa y bien establecida. Esto es especialmente cierto, si cabe, en el caso de la psicología, debido a la naturaleza de su objeto de estudio, en muchos aspectos complejo. El ámbito de la logoterapia, desarrollada por Viktor Emil Frankl, es un ejemplo de la dificultad que supone encontrar instrumentos de medida válidos y fiables. Frankl (1994) afirma que la motivación humana prioritaria es percibir y experimentar que la vida tiene sentido, y enfatiza el papel de la autotrascendencia y de los valores creativos, experienciales y actitudinales en el desarrollo del sentido de plenitud existencial. Cuando la voluntad de sentido no se satisface, se experimenta el vacío existencial, un tipo específico de neurosis, que Frankl denomina "noógena" (Frankl, 1997, 2003), relacionada con abuso de sustancias, ideación suicida y desesperanza (p. ej., García-Alandete, Gallego-Pérez \& Pérez-Delgado, 2009; Guibert \& Del Cueto, 2003).

El Purpose-In-Life Test ([PIL]; Crumbaugh \& Maholic, 1969) es el instrumento hoy por hoy más utilizado a efectos de investigación sobre el sentido de la vida, desde supuestos logoterapéuticos, concretamente su Parte A, una escala de 20 ítems tipo Likert, con 7 categorías de respuesta (ver Apéndice). De hecho, su construcción y validación supuso un impulso de primer orden en la investigación empírica sobre un constructo, el de «sentido de la vida», hasta entonces más propio de la reflexión filosófica y, en el campo de la psicología, limitado al ámbito de lo humanístico-existencial, caracterizado más por la teorización que por la operativización empírica y la construcción de instrumentos de evaluación. En la actualidad, el sentido de la vida es un constructo empíricamente fundamentado y objeto de investigación pluridisciplinar. Un aspecto particular de interés recurrente en relación con este instrumento es el de su estructura factorial, pues es notable la disparidad de procedimientos y resultados entre distintos trabajos.

Noblejas de la Flor (1994), con una submuestra no clínica muy heterogénea formada por 823 españoles, obtuvo cuatro factores, que explicaron el $54 \%$ del total de la varianza: Percepción de sentido (items 4, 6, 9-12, 16, 17 y 20; 35.9\% de la varianza); Experiencia de sentido (ítems 1, 2, 5, 9, 17, 19 y 20; $6.8 \%$ de la varianza); Metas y tareas (ítems 3, 7, $8,13,17,19$ y $20 ; 5.8 \%$ de la varianza) y Dialéctica destino/libertad (ítems 14, 15 y 18; $5.5 \%$ de la varianza). En el citado trabajo no se impusieron requisitos exigentes para la reducción de la escala (únicamente, que los ítems debían mostrar un valor de saturación mínimo de 0.3), aceptándose ítems que saturaban en más de un factor: el 9 saturó en Percepción de sentido y Experiencia de sentido, el 17 saturó en Percepción de sentido, Experiencia de sentido y Metas y tareas, el 19 saturó en los factores Experiencia de sentido y Metas y tareas y el 20 saturó en los tres factores mencionados. Los ítems señalados, en consecuencia, tenían una baja validez discriminante y su aceptación constituyó un aspecto metodológicamente crítico en el proceso de reducción factorial de la escala.

Magaña, Zavala, Ibarra, Gómez y Gómez (2004), con una muestra de 723 mexicanos de edades entre los 16 y los 27 años, obtuvieron alta consistencia interna del PIL, $\alpha=0.9$, y mediante análisis factorial exploratorio, un modelo trifactorial que explicó el $50.06 \%$ de la varianza: Percepción de sentido y Significado de la vida (ítems 1-5, 7-10, 13, 17, 19 y $20 ; 24.84 \%$ de la varianza), Satisfacción por la propia vida (ítems $6,11,12,16$ y $18 ; 17.26 \%$ de la varianza) y Libertad y control de la propia vida (ítems 14 y 15; $7.96 \%$ de la varianza).

Risco (2009) con una muestra española de 1.151 preuniversitarios de 13 a 17 años y 1.173 universitarios de 18 a 25 años (61.2 \% del total de la muestra eran mujeres) obtuvo una estructura trifactorial que explicaba el $40.99 \%$ de la varianza total: Valor de la vida (ítems 1, 4, 6, 9-12, ; $24.71 \%$ de la varianza), Capacidad de significado (ítems 2 , $5,7,14,15,17-19 ; 10.07 \%$ de la varianza) y Metas y responsabilidad (ítems 3, 8, 13 y 20; $6.45 \%$ de la varianza). Además, obtuvo un alfa de Cronbach de 0.81 para la escala total.

Schulenberg y Melton (2010) realizaron un estudio en el que compararon varios de los modelos propuestos en la literatura para el PIL, mediante análisis factorial confirmatorio, obteniendo para 
el bifactorial de Morgan y Farsides (2009) el ajuste más adecuado (Tabla 1).

Schulenberg y Melton (2010), con una muestra de 620 universitarios norteamericanos, $M_{\text {edad }}=19.7$, contrastaron los modelos incluidos en la Tabla 1, obteniendo apoyo empírico para el modelo bifactorial de Morgan y Farsides (2009), el cual se extrajo de un estudio en el que se utilizó una muestra heterogénea con edades entre los 15 y los 75 años, $M_{\text {edad }}=32, D E=12.35$.

Jonsén et al. (2010), con una muestra de 449 suecos (278 mujeres y 166 hombres entre 19 y 103 años), obtuvieron una estructura trifactorial y una consistencia interna (alfa de Cronbach) para el total de la escala de 0.83. La consistencia interna fue de $0.83,0.64$ y 0.54 , respectivamente, para los factores Sentido de la existencia (ítems 1, 3, 4, 6, $8,9,11$ y $20 ; 26.9 \%$ de la varianza), Libertad para crear sentido en la vida cotidiana (ítems 10, 14, 15, $17-19 ; 7.7 \%$ de la varianza) y Voluntad para encontrar sentido ante retos futuros (ítems 2, 5 y 7; $6.6 \%$ de la varianza). Los ítems 12,13 y 16 no cargaron sobre ningún factor.

Como se desprende de lo expuesto, los estudios sobre la estructura factorial del PIL son heterogéneos en cuanto a muestra utilizada, tipo de análisis, criterios para interpretar los factores y resultados. Estas divergencias sugieren la conveniencia de un análisis de la estructura factorial del PIL más riguroso, ya que se trata del instrumento más utilizado en la investigación empírica sobre el sentido de la vida desde los supuestos de la logoterapia. Con muestra española, el trabajo de referencia para la estructura factorial del PIL es el de Noblejas de la Flor (1994), que utilizó una muestra muy heterogénea y no impuso criterios al proceso de reducción de la escala, haciendo conveniente una revisión de la estructura factorial de este instrumento. Debe añadirse que, mientras que Noblejas de la Flor obtiene cuatro factores, los trabajos más representativos de la literatura al respecto obtienen uno o dos factores; de hecho, como se ha señalado más arriba, los análisis confirmatorios de Schulenberg y Melton (2010) apoyaban el modelo bifactorial con reducción de la escala de Morgan y Farsides (2009) a 10 ítems.

Dada la relevancia de la validez estructural de cualquier escala de medida, parece esencial que la incertidumbre con respecto a la dimensionalidad del PIL sea resuelta. Y esto a pesar de que puede ser cierto, como afirman Schulenberg y Melton (2010), que el gran número de modelos desarrollados con el análisis factorial exploratorio resulta en una literatura complicada con respecto a la validez factorial del PIL.

El objetivo del presente trabajo fue analizar la estructura factorial del PIL. Puesto que existe divergencia en cuanto al número y composición de sus factores, se procedió, en primer lugar, a un Análisis Factorial Exploratorio (AFE), sin asumir supuestos a priori sobre la estructura de la escala -dada la divergencia entre los estudios previos, desde modelos unifactoriales a tetrafactoriales-. Posteriormente, se llevó a cabo un Análisis Factorial Confirmatorio (AFC) de la escala resultante.

\section{Método}

\section{Participantes}

Participaron voluntariamente 457 universitarios (320 mujeres, $70 \%$; 137 hombres, $30 \%$ ), estudiantes de psicología en Valencia (España), con un rango de edades entre 18 y 55 años, $M=21.8, D E=4.56$. El muestreo fue incidental, y no obtuvieron incentivo académico alguno por su colaboración.

\section{Instrumento}

Se utilizó la versión española de la Parte A del Purpose-in-Life Test ([PIL]; Crumbaugh \& Maholick, 1964, 1969) realizada por Noblejas de la Flor (1994), ya descrita más arriba en sus aspectos psicométricos básicos. Se trata de una escala compuesta por 20 ítems tipo Likert, con categorías de respuesta de 1 a 7 (Tabla 2). Las categorías 1 y 7 tienen anclajes de respuesta específicos para el enunciado de cada ítem, y la categoría 4 representa una posición neutra.

\section{Procedimiento y análisis estadísticos}

La cumplimentación del protocolo que incluía la versión española del PIL se llevó a cabo en las aulas 


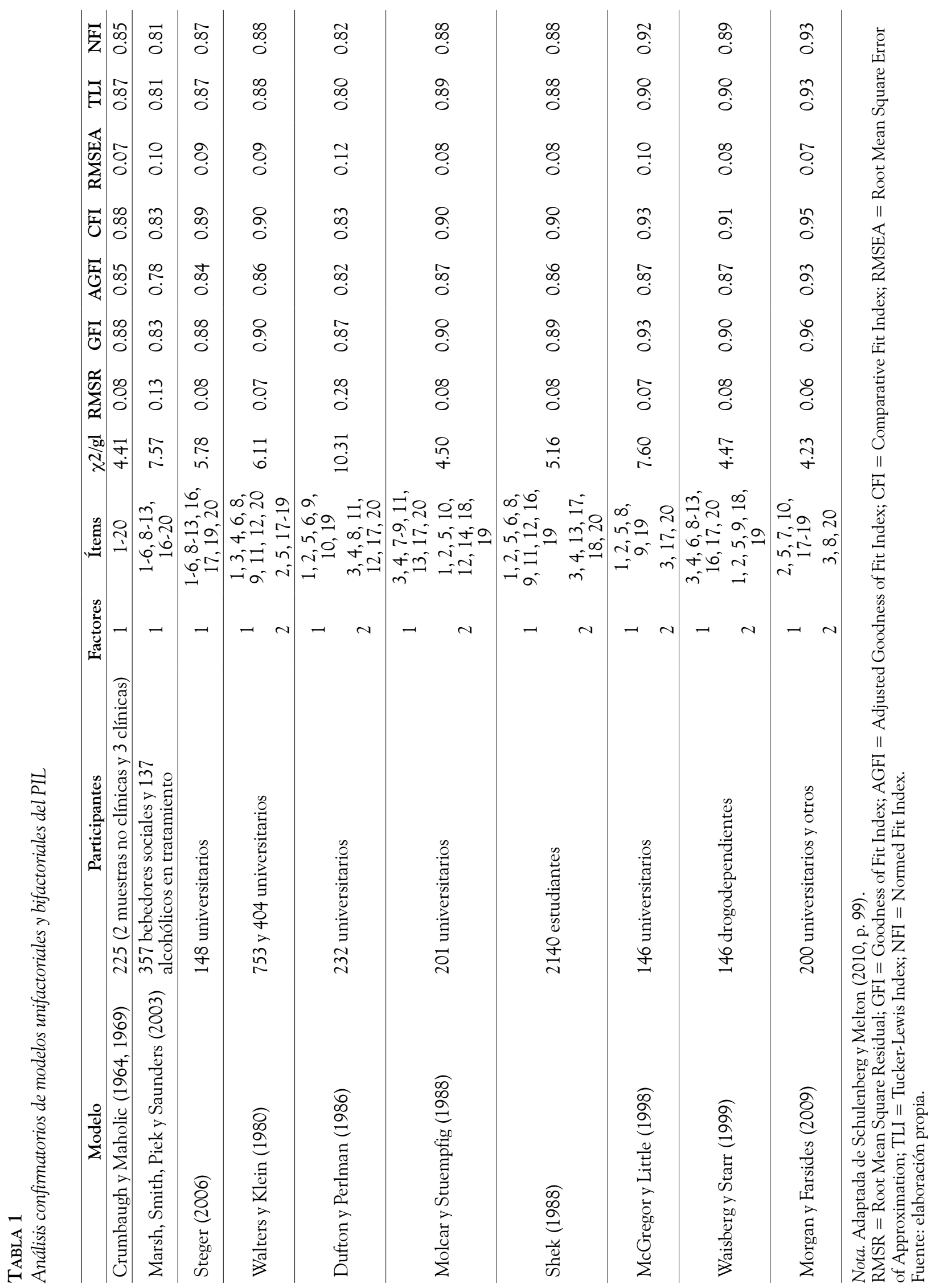


en las que de ordinario los participantes desarrollaban su actividad académica, en horario lectivo y bajo la supervisión de los autores. Se aseguró el anonimato y la confidencialidad de los resultados, se resolvieron las dudas relativas al procedimiento y se insistió en la sinceridad de las respuestas, para maximizar la validez de la información obtenida. El tiempo promedio de cumplimentación del protocolo fue de 15 minutos.

Se llevó a cabo un Análisis de Componentes Principales (ACP) con rotación Varimax (Nunnally \& Bernstein, 1994; Rennie, 1997) y normalización de Kaiser, que es el análisis más frecuente en los trabajos revisados, como se ha señalado más arriba. El empleo de este método de rotación estuvo motivado en que no se partía de una concepción a priori de la estructura factorial de la escala -unifactorial según sus constructores (Crumbaugh \& Maholic, 1969)-, por lo que no se presupuso que pudieran existir varios factores correlacionados, y en que se pretendía que la solución obtenida fuera lo más simple y estable posible.

Además de los criterios relativos a las pruebas $\mathrm{KMO}$, de esfericidad de Bartlett y autovalor superior a 1 , se consideraron los siguientes criterios para la depuración de la escala: (1) cada factor debía explicar al menos el $5 \%$ de la varianza total, (2) la carga factorial de los ítems en los factores debía ser igual o superior a 0.5, (3) los ítems debían saturar en un solo factor y (4) los factores debían presentar una consistencia interna (alfa de Cronbach) superior a 0.7 (Nunnally \& Bernstein, 1994). El ACP se realizó con el programa SPSS 17.0 para Windows y para comprobar la bondad del ajuste datos-modelo se llevó a cabo un AFC con el programa EQS 6.1 para Windows.

\section{Resultados}

\section{Análisis Factorial Exploratorio}

Las puntuaciones medias de los ítems del PIL fueron muy altas, considerando que el rango de respuesta es de 1 a 7 (Tabla 2). La media más baja fue la del ítem $15, M=4.28$, relativo a la preocupación y temor ante la muerte, haciendo pensar que es un ítem al que se responde sesgadamente, ya que difícilmente se está preparado y sin temor ante la muerte en población como la de la muestra, compuesta por estudiantes universitarios con una edad media de 21.8 años. En el mismo rango de puntuación media, en torno al punto 4 , se hallaron los ítems 12 , $M=4.52$, relativos a la confusión/adaptación con que se percibe el mundo en relación con la propia vida y $14, M=4.85$, relativo a determinismo ambiental/hereditario vs. libertad personal para la toma de decisiones. Es decir, las medias más bajas (que, con todo, no son bajas en términos relativos) se relacionaron con preparación vs. temor ante la muerte, confusión vs. adaptación entre mundo y vida propia, y determinismo vs. libertad. El resto de ítems superaron la puntuación media de 5, alcanzando algunos incluso una media superior a 6 (ítems 3 y 7). Por otra parte, todos los ítems correlacionaron positiva y significativamente con la puntuación total de la escala.

Se procedió al proceso de reducción de la escala mediante ACP con método de rotación Varimax, aplicándose rigurosamente los criterios de depuración de la escala, hasta que se obtuvo una solución terminal satisfactoria (Tabla 3). En todos los casos, el estadístico KMO y el test de Bartlett indicaron que se podía aplicar el análisis factorial.

El resultado fue una escala de 10 ítems con alta consistencia interna, $\alpha=0.862$ y con dos factores de primer orden (Tabla 4). Al Factor 1 (ítems 1, 2, $5,6,9$ y 11) lo denominamos Satisfacción y Sentido de la Vida (SSV) y al Factor 2 (ítems 3, 7, 17 y 20) lo denominamos Metas y Propósitos Vitales (MPV). Un análisis de correlación mostró que ambos factores se hallaban significativamente correlacionados, $r=0.582, p<0.01$, dato que se tuvo en consideración en el Análisis Factorial Confirmatorio.

\section{Análisis Factorial Confirmatorio}

Se procedió a un AFC del modelo obtenido mediante el análisis exploratorio. El valor de la estimación normalizada de la curtosis multivariada, coeficiente de Mardia $=41.7828$, sugirió el uso del método de máxima verosimilitud con estimación robusta (Bentler, 2006). El estadístico chi cuadrado 
TABLA 2

Estadísticos descriptivos de los items del PIL y correlaciones con la escala

\begin{tabular}{|c|c|c|c|}
\hline Ítems del PIL & M & DT & $\begin{array}{l}\text { r (item- } \\
\text { escala) }\end{array}$ \\
\hline Generalmente me encuentro: Completamente aburrido/Exuberante, entusiasmado & 5.11 & 1.08 & $0.59 * *$ \\
\hline La vida me parece: Completamente rutinaria/Siempre emocionante & 5.17 & 1.27 & $0.67 * *$ \\
\hline En la vida tengo: Ninguna meta o anhelo/Muchas metas y anhelos definidos & 6.27 & 0.86 & $0.52 * *$ \\
\hline Mi existencia personal es: Sin sentido ni propósito/Llena de sentidos y propósitos & 5.96 & 1.07 & $0.72 * *$ \\
\hline Cada día es: Exactamente igual/Siempre nuevo y diferente & 5.24 & 1.36 & $0.66 * *$ \\
\hline Si pudiera elegir: Nunca habría nacido/Tendría otras nueve vidas iguales a esta & 5.66 & 1.17 & $0.63 * *$ \\
\hline $\begin{array}{l}\text { Después de retirarme: Holgazanearía el resto de mi vida/Haría las cosas emocionantes que siem- } \\
\text { pre deseé realizar }\end{array}$ & 6.25 & 1.01 & $0.37 * *$ \\
\hline $\begin{array}{l}\text { En el logro de mis metas vitales: No he conseguido ningún progreso/He llegado a mi realización } \\
\text { completa }\end{array}$ & 5.11 & 0.95 & $0.62 * *$ \\
\hline Mi vida es: Vacía y llena de desesperación/Un conjunto de cosas buenas y emocionantes & 5.65 & 1.10 & $0.79 * *$ \\
\hline Si muriera hoy, me parecería que mi & 5.71 & 1.32 & $0.71 * *$ \\
\hline $\begin{array}{l}\text { i propia vida: Me pregunto a menudo la razón por la que existo/Siempre encuen- } \\
\text { vivir }\end{array}$ & 5.76 & 1.55 & 0.74 \\
\hline 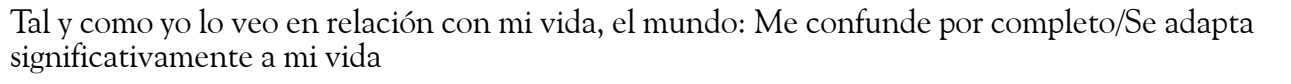 & 4.52 & 1.4 & 0.56 \\
\hline Me considero: Una persona irresponsable/Una persona muy responsable & 5.72 & 1.20 & $0.43 * *$ \\
\hline $\begin{array}{l}\text { Con respecto a la libertad de que dispone para hacer sus propias elecciones, creo que el hombre } \\
\text { es: Completamente esclavo de las limitaciones de la herencia y del ambiente/Absolutamente } \\
\text { libre de hacer todas sus elecciones vitales }\end{array}$ & 4.85 & 1.56 & $0.52 * *$ \\
\hline Con respecto a la muerte, estoy: Falto de preparación y atemorizado/Preparado y sin temor & 4.28 & 1.74 & $0.29 * *$ \\
\hline ado seriamente como una salida a mi situación/Nunca & 5.88 & 1.46 & 0.51 \\
\hline contrar un significado, un propósito o una misión en la vida & 5.92 & 1.03 & 0.612 \\
\hline $\begin{array}{l}\text { Mi vida está: Fuera de mis manos y controlada por factores externos/En mis manos y bajo mi } \\
\text { control }\end{array}$ & 5.36 & 1.20 & 0.56 \\
\hline $\begin{array}{l}\text { Enfrentarme a mis tareas cotidianas supone: Una experiencia dolorosa y aburrida/Una fuente de } \\
\text { placer y satisfacción }\end{array}$ & 5.13 & 1.13 & $0.697 * *$ \\
\hline $\begin{array}{l}\text { He descubierto: Ninguna misión o propósito en mi vida/Metas claras y un propósito satisfacto- } \\
\text { rio para mi vida }\end{array}$ & 5.90 & 1.00 & $0.691 * *$ \\
\hline
\end{tabular}

Nota. $* * p<0.01$ (bilateral).

Fuente: elaboración propia.

Satorra-Bentler, $\chi_{\mathrm{SB}}^{2}=101.0105, g l=34, p<0.01$, sugirió el uso de los índices de ajuste, que fueron adecuados: $\mathrm{NFI}=0.898, \mathrm{NNFI}=0.907, \mathrm{CFI}=$ $0.929, \mathrm{IFI}=0.930, \mathrm{MFI}=0.929, \mathrm{RMSEA}=0.066$ [0.051, 0.080]. El índice NFI fue solo ligeramente inferior al 0.9 recomendado, superando este valor el resto de índices de ajuste y parsimonia. El índice de penalización RMSEA fue inferior a 0.08 , valor que marca el límite de aceptabilidad (Bentler \& Bonnet, 1980; Browne \& Cudeck, 1993; Hair, Anderson, Tatham \& Black, 2007).
Dado que el AFC confirmó la alta correlación interfactorial, $r=0.734, p<0.05$, siguiendo el procedimiento de otros trabajos (p. ej., Arribas, 2013) se procedió a estimar un modelo con un factor de segundo orden, el cual, si bien mostró índices de ajuste mejores que el de dos factores correlaciona$\operatorname{dos}, \chi_{\mathrm{SB}}^{2}=73.9115, g l=33, p<0.01 ; \mathrm{NFI}=0.926$, $\mathrm{NNFI}=0.941, \mathrm{CFI}=0.957$, IFI $=0.957, \mathrm{MFI}=$ 0.956, RMSEA $=0.052[0.036,0.068]$, presentó estimaciones infractoras en la ecuación estandarizada que sugerían su inadecuación (Hair et al., 2007), 
TABLA 3

Análisis de Componentes Principales realizados hasta obtener la solución terminal

\begin{tabular}{|c|c|c|c|c|c|c|}
\hline $\mathrm{ACP}$ & Factor & Ítems & $\begin{array}{l}\text { \% varianza } \\
\text { explicada }\end{array}$ & $\begin{array}{l}\text { alfa de } \\
\text { Cronbach }\end{array}$ & $\begin{array}{l}\text { \% total varianza } \\
\text { explicada }\end{array}$ & Observaciones \\
\hline \multirow{4}{*}{$1^{\mathrm{o}}$} & 1 & $1,2,4,5,6,9$ & 19.720 & 0.856 & \multirow{4}{*}{56.129} & \multirow{4}{*}{$\begin{array}{l}\text { Ítems } 8,10 \text { y } 13 \text { no alcanzaron valor de } \\
\text { saturación mínimo } \\
\text { Rechazo del Factor } 4\end{array}$} \\
\hline & 2 & $11,12,14,18,19$ & 15.315 & 0.748 & & \\
\hline & 3 & $3,7,17,20$ & 14.799 & 0.71 & & \\
\hline & 4 & $15(-), 16$ & 6.294 & 0.042 & & \\
\hline \multirow{3}{*}{$2^{\circ}$} & 1 & $1,2,4,5,6,9,11$ & 24.434 & 0.868 & \multirow{3}{*}{57.546} & \multirow{3}{*}{$\begin{array}{l}\text { Rechazo del Factor } 3 \text { (no alcanzó } \alpha \\
\text { mínimo) }\end{array}$} \\
\hline & 2 & $3,7,17,20$ & 16.808 & 0.71 & & \\
\hline & 3 & $12,14,18,19$ & 16.304 & 0.698 & & \\
\hline \multirow{2}{*}{$3^{\circ}$} & 1 & $1,2,4,5,6,9,11$ & 34.047 & 0.868 & \multirow{2}{*}{57.264} & \multirow{2}{*}{$\begin{array}{l}\text { Rechazo del ítem } 4 \text { (saturó en ambos } \\
\text { factores) }\end{array}$} \\
\hline & 2 & $3,4,7,17,20$ & 23.217 & 0.71 & & \\
\hline \multirow{2}{*}{$4^{\circ}$} & 1 & $1,2,5,6,9,11$ & 34.172 & 0.848 & \multirow{2}{*}{57.273} & \multirow{2}{*}{ El modelo cumplió todos los requisitos } \\
\hline & 2 & $3,7,17,20$ & 23.101 & 0.71 & & \\
\hline
\end{tabular}

Fuente: elaboración propia.

en concreto, un coeficiente estandarizado igual a la unidad en la ecuación de saturación del factor Metas y Propósitos Vitales en el factor de segundo orden (Tabla 5). Como señala Conchado (2011, p. 264), "en el caso de los coeficientes estandarizados muy próximos a la unidad el investigador debe valorar la posibilidad de eliminar uno de los constructos asociados a dichos coeficientes". El contraste de Wald (1943) confirmó la inadecuación de este parámetro, aconsejando su eliminación.

En consecuencia, el modelo con un factor de segundo orden fue desechado y fue aceptado el modelo de dos factores correlacionados que se obtuvo inicialmente. La estructura de la escala resultante, que denominamos Purpose In Life Test-10 (PIL-10), queda por tanto conformada por los dos factores

\section{TABLA 4}

Matriz de componentes rotados ${ }^{(a)}$

\begin{tabular}{lcc}
\hline \multicolumn{1}{c}{ Ítem del PIL } & Componente \\
\cline { 2 - 2 } & \multicolumn{1}{c}{1} & \multicolumn{1}{c}{2} \\
\hline 2. La vida me parece: Completamente rutinaria/Siempre emocionante & 0.842 \\
5. Cada día es: Exactamente igual/Siempre nuevo y diferente & 0.769 \\
9. Mi vida es: Vacía y llena de desesperación/Un conjunto de cosas buenas y emocionantes & 0.741 \\
1. Generalmente me encuentro: Completamente aburrido/Exuberante, entusiasmado & 0.717 \\
11. Al pensar en mi propia vida: Me pregunto a menudo la razón por la que existo/Siempre encuen- & 0.659 \\
tro razones para vivir & 0.551 \\
6. Si pudiera elegir: Nunca habría nacido/Tendría otras nueve vidas iguales a esta & \\
7. Después de retirarme: Holgazanearía el resto de mi vida/Haría las cosas emocionantes que siem- & \\
pre deseé realizar & \\
20. He descubierto: Ninguna misión o propósito en mi vida/Metas claras y un propósito satisfacto- \\
rio para mi vida & 0.692 \\
3. En la vida tengo: Ninguna meta o anhelo/Muchas metas y anhelos definidos & 0.667 \\
17. Considero que mi capacidad para encontrar un significado, un propósito o una misión en la vida \\
es: Prácticamente nula/Muy grande
\end{tabular}

Nota. Método de extracción: Análisis de Componentes Principales. Método de rotación: Varimax con Normalización de Kaiser. (a) La rotación convergió en 3 iteraciones.

Fuente: elaboración propia. 
TABLA 5

Solución estandarizada del modelo con un factor de segundo orden

\begin{tabular}{cccc}
\hline & $\begin{array}{c}\text { Coeficiente } \\
\text { estandarizado }\end{array}$ & $\begin{array}{c}\text { Varianza } \\
\text { de error }\end{array}$ & $\mathrm{R}^{2}$ \\
\hline Ítem 1 & $0.661^{*} \mathrm{~F} 1$ & $0.750 \mathrm{E} 1$ & 0.437 \\
Ítem 2 & $0.763^{*} \mathrm{~F} 1$ & $0.647 \mathrm{E} 2$ & 0.582 \\
Ítem 3 & $0.644^{*} \mathrm{~F} 2$ & $0.765 \mathrm{E} 3$ & 0.414 \\
Ítem 5 & $0.704^{*} \mathrm{~F} 1$ & $0.710 \mathrm{E} 5$ & 0.496 \\
Ítem 6 & $0.646^{*} \mathrm{~F} 1$ & $0.763 \mathrm{E} 6$ & 0.417 \\
Ítem 7 & $0.386^{*} \mathrm{~F} 2$ & $0.923 \mathrm{E} 7$ & 0.149 \\
Ítem 9 & $0.786^{*} \mathrm{~F} 1$ & $0.619 \mathrm{E} 9$ & 0.617 \\
Ítem 11 & $0.665^{*} \mathrm{~F} 1$ & $0.747 \mathrm{E} 11$ & 0.442 \\
Ítem 17 & $0.657^{*} \mathrm{~F} 2$ & $0.753 \mathrm{E} 17$ & 0.432 \\
Ítem 20 & $0.804^{*} \mathrm{~F} 2$ & $0.594 \mathrm{E} 20$ & 0.647 \\
F1 & $0.734^{*} \mathrm{~F} 3$ & $0.680 \mathrm{D} 1$ & 0.538 \\
F2 & $1.000^{*} \mathrm{~F} 3$ & $0.000 \mathrm{D} 2$ & 1.000 \\
\hline
\end{tabular}

Nota. F1 = factor SSV, F2 = factor MPV, F3 = factor de segundo orden. $* p<0.05$.

Fuente: elaboración propia. de primer orden correlacionados, Satisfacción y Sentido de la Vida ([SSV]; ítems 1, 2, 5, 6, 9 y 11 de la escala original) y Metas y Propósitos Vitales ([MPV]; ítems 3, 7, 17 y 20 de la escala original). La Figura 1 muestra la solución estandarizada de este modelo. Los coeficientes estandarizaros se indican sobre las líneas que unen los ítems con los factores, y los coeficientes de determinación, $R^{2}$, que indican el porcentaje de varianza explicada, se muestran sobre los rectángulos de los ítems.

Todos los parámetros de la ecuación estandarizada presentaron valores aceptables. Para el factor SSV, el ítem que explicó el mayor porcentaje de varianza fue el 9, seguido de los ítems 2, 5, 11, 1 y 6. Para el factor MPV, el ítem que explicó mayor porcentaje de varianza fue el 20 , seguido del 17 , el 3 y el 7. El factor SSV está relacionado con la percepción y experiencia del sentido de la vida, y con la satisfacción personal con la misma; tiene,

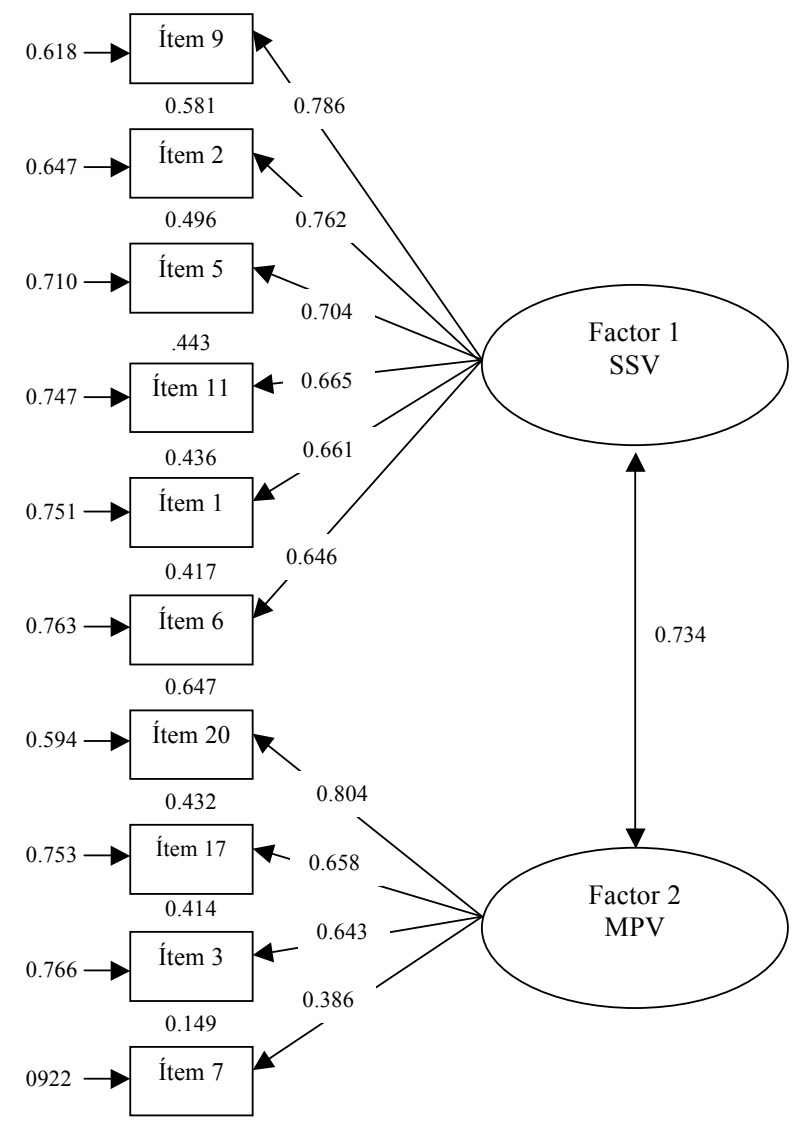

Figura 1. Solución estandarizada del modelo de dos factores correlacionados Fuente: elaboración propia 
pues, un carácter fundamentalmente cognitivoevaluativo. El factor MPV está relacionado con el hecho de tener definida una misión u objetivos en la vida, hacia cuyo logro la persona orienta sus esfuerzos y actividades; parece tener, por tanto, un carácter más marcadamente motivacional. La escala, por tanto, incluye ítems que miden los aspectos cognitivo-evaluativo y motivacional relacionados con el sentido de la vida.

Diferencias en función del género y de la edad

La puntuación media de las mujeres fue superior a la de los hombres en la escala, $M=57.39$, $D E=7.84$ y $M=55.8, D E=7.45$, respectivamente; en el factor SSV, $M=32.76, \mathrm{DE}=5.96$ y $M=32.17, D E=5.13$, respectivamente; $y$ en el factor $M P V, M=24.64, D E=2.77$ y $M=23.63$, $D E=2.95$, respectivamente, siendo las diferencias estadísticamente significativas en la escala, $t(455)=$ 2.027, $p=0.043$, y en el factor MPV, $t(455)=3.5$, $p=0.001$, pero no en el factor SSV, $t(455)=1.01$, $p=0.315$.

Para el análisis de las diferencias en función de la edad, la prueba de Kolmogorov-Smirnov, $Z=4.98, p<0.01$, sugirió el uso de estadísticos no paramétricos, indicando la prueba de KruskalWallis diferencias no significativas ni en el caso de la escala, $\chi^{2}(25)=24.25, p=0.505$, ni de los factores SSV, $\chi^{2}(25)=24.88, p=0.469$, y MPV, $\chi^{2}(25)=25.53, p=0.433$.

\section{Discusión}

El objetivo principal del presente trabajo fue examinar la estructura factorial y la consistencia interna del Purpose-In-Life Test ([PIL]; Crumbaugh \& Maholic, 1969). Para esto se utilizó la versión española de Noblejas de la Flor (1994) y se llevó a cabo un Análisis de Componentes Principales (ACP), siendo el modelo resultante contrastado mediante Análisis Factorial Confirmatorio (AFC). Adicionalmente, se ofrecen datos sobre los estadísticos descriptivos de la escala y las diferencias en las puntuaciones total y factoriales asociadas al género y la edad.
Para el ACP, se impusieron restricciones exigentes en cuanto a porcentaje que debían explicar los factores, saturación de los ítems, cantidad de ítems en cada factor y consistencia interna de los factores. Estos requisitos tan exigentes se justifican por la divergencia sobre la estructura factorial del PIL en la investigación previa. El proceso de reducción dio lugar a una escala de 10 ítems (de los 20 originales), con una estructura de dos factores correlacionados que explicó el 57.273 \% de la varianza total. Los factores fueron denominados Satisfacción y Sentido de la Vida ([SSV]; ítems 1, 2, 5, 6, 9 y 11; 34.172\% de la varianza) y Metas y Propósitos en la Vida ([MPV]; ítems 3, 7, 17 y 20; $23.101 \%$ de la varianza). La estructura factorial obtenida coincide con algunos de los modelos analizados confirmatoriamente por Schulenberg y Melton (2010), concretamente con Walters y Klein (1980), Dufton y Perlman (1986), Molcar y Stuempfig (1988), Shek (1988), McGregor y Little (1998), Waisberg y Starr (1999) y Morgan y Farsides (2009), que mostraban mejor ajuste que los modelos unifactoriales (Crumbaugh \& Maholic, 1969; Marsh et al., 2003; Steger, 2006).

El coeficiente alfa obtenido por la escala indica una alta consistencia interna, $\alpha=0.862$, similar a la de trabajos precedentes (p. ej., Jonsén et al., 2010; Magaña et al., 2004; Melton \& Schulenberg, 2007, 2008; Noblejas de la Flor, 2000; Nygren et al., 2005; Reker, 2000; Schulenberg, 2004). El factor SSV mostró una alta consistencia interna, $\alpha=0.848$, y la del factor MPV fue aceptable, $\alpha=0.71$. Estos valores permiten concluir que, tanto globalmente como en cada una de sus factores, la escala presenta una alta homogeneidad de sus ítems.

La reducción de la escala a 10 ítems, en lugar de los 20 ítems de la versión original (Crumbaugh \& Maholic, 1969) y de la adaptación española utilizada (Noblejas de la Flor, 1994), supone una simplificación de la estructura factorial del PIL que redunda en una mejora sustancial de la parsimonia del instrumento, reduciéndose a un modelo de dos factores correlacionados con un buen ajuste, como muestra el AFC: un factor cognitivo-evaluativo, relacionado con la percepción y valoración general del sentido de la vida (SSV) y un factor motivacional, relacionado con el establecimiento de metas y 
propósitos vitales concretos (MPV). Tener metas y propósitos vitales, percibir la vida como un proyecto orientado a su consecución, estar siempre dispuesto a alcanzar objetivos y conseguir logros, y experimentar que la vida está llena de sentido, es valiosa y excitante, son aspectos del sentido de la vida estrechamente correlacionados entre sí: a más propósito de metas, mayor satisfacción vital, y viceversa.

Aunque se observan coincidencias notables con todos los modelos bifactoriales revisados, el obtenido en este trabajo coincide en mayor medida con el de Shek (1988): en ambos factores este autor incluye los mismos ítems, si bien en el Factor 1 incluye también los ítems 8, 12, 16 y 19 y en el Factor 2 los ítems 4, 13 y 18 (eliminados en el proceso reductivo llevado a cabo en este trabajo), y falta el ítem 7 en el Factor 2 (mantenido en la versión obtenida en este trabajo). Llama la atención que este último ítem está ausente en los modelos de Walters y Klein (1980), Dufton y Perlman (1986), Shek (1988), McGregor y Little (1998) y Waisberg y Starr (1999). Posiblemente, las razones para estas diferencias son metodológicas, relacionadas con los distintos requisitos exigidos para la reducción de la escala.

La edad no dio lugar a diferencias estadísticamente significativas en la escala obtenida. En cuanto a las diferencias en función del género, las mujeres alcanzaron significativamente puntuaciones más altas que los hombres en la puntuación total y en el factor MPV. Al respecto, los resultados de otros trabajos son dispares (si bien usando una versión del PIL distinta a la resultante en el presente trabajo): algunos obtienen diferencias no significativas (Crumbaugh \& Maholick, 1964; Gallego-Pérez \& García-Alandete, 2004; García-Alandete et al., 2009; Jackson \& Coursey, 1988; Meier \& Edwards, 1974; Noblejas de la Flor, 1994; Preble, 1986; Robbins \& Francis 2005; Sallee \& Casciani, 1976), otros que las mujeres alcanzan puntuaciones significativamente más altas (Crumbaugh, 1968; Doerries, 1970; García-Alandete, Rosa, Soucase \& Gallego-Pérez, 2011; Molasso, 2006; Nygren et al., 2005) y algún trabajo en el que las puntuaciones más altas son alcanzadas por los hombres (Nygren et al., 2005) .
Hay que indicar que estas investigaciones se han centrado en la puntuación total del PIL, dejando fuera de análisis sus factores (Noblejas de la Flor, 1994; Reker, 2000; Reker \& Chamberlain, 2000), a excepción del trabajo de García-Alandete et al. (2011) con la versión tetrafactorial de 20 ítems de Noblejas de la Flor (1994), en los cuales las mujeres alcanzaron puntuaciones más altas que los hombres en la puntuación total del PIL y en los factores Percepción de sentido (ítems 4, 6, 9-12, 16-17 y 20) y Metas y tareas (ítems 3, 7-8, 13, 17, 19 y 20), relativos, respectivamente, a los motivos y razones para vivir la vida y la valoración que esta merece, y a los objetivos ligados a acciones concretas en la vida y la responsabilidad personal percibida hacia los mismos. En el factor Metas y Tareas del modelo de Noblejas de la Flor (1994) se incluyen los ítems que en el presente trabajo han saturado en el factor Metas y Propósitos en la Vida (ítems 3, 7, 17 y 20; los ítems 8,13 y 19 han sido desestimados en el proceso de reducción, debido al empleo de criterios más restrictivos). Al respecto, García-Alandete et al. (2011) hipotetizan la existencia de diferencias cognitivas básicas, concretamente organizativo-perceptuales, entre mujeres y hombres que operarían sobre la capacidad de percibir y atribuir sentido al ambiente, de interpretar y organizar los estímulos con el fin de construir totalidades llenas de sentido. Como se ha señalado, en el presente trabajo las mujeres puntuaron más alto en el total y en el factor MPV de la versión obtenida del PIL; la explicación está condicionada a futuras investigaciones específicas, resultando muy sugerente la hipótesis de las diferencias organizativo-perceptuales que plantean los citados autores.

Debe resaltarse que sobre las propiedades psicométricas del PIL con población española solo se cuenta con los precedentes de Noblejas de la Flor (1994) y de Risco (2009), en los que se obtuvieron estructuras factoriales muy alejadas de los modelos analizados por Schulenberg y Melton (2010) y de la obtenida en este trabajo. De manera más concreta y a modo de ejemplo, el trabajo de Noblejas de la Flor (1994) no estableció criterios restrictivos a sus datos para el proceso de reducción de la escala, aceptando saturaciones de varios ítems en más de un factor 
(los ítems 17 y 20 saturaron en los Factores 1, 2 y 3; el ítem 9, en los Factores 1 y 2; el ítem 19, en los Factores 2 y 3), dando lugar a una estructura factorial con baja validez discriminante y exigiendo una revisión del proceso de reducción con criterios más exigentes, como la realizada en el presente trabajo.

Ciertamente, los resultados de este trabajo deben ser interpretados a la luz de ciertas limitaciones, sin menoscabo de sus aportaciones. En primer lugar, si bien el rango de edades del grupo de participantes está entre 18 y 55 años, en su mayor parte está compuesto por jóvenes, $M=21.8$, no siendo representativo de la población general. En segundo lugar, el número de mujeres es sensiblemente superior al de hombres; no obstante, la proporción es similar a la de trabajos previos.

Debe añadirse el interés de realizar estudios psicométricos del PIL con población clínica, pues posiblemente algunos de los ítems de la escala original se mantendrían en un análisis factorial. Por ejemplo, el ítem 15 («Con respecto a la muerte estoy: Falto de preparación y atemorizado / Preparado y sin temor») o el ítem 16 («Con respecto al suicidio: Lo he considerado seriamente como una salida a mi situación / Nunca le he dedicado un segundo pensamiento») pueden ser relevantes para ciertas poblaciones específicas (p. ej., personas con enfermedad en fase terminal, con dolor crónico o con grave discapacidad, de edad avanzada, con trastorno de personalidad o depresión, entre otras).

Para finalizar, los resultados obtenidos en este trabajo evidencian la conveniencia de clarificar las propiedades psicométricas del PIL, especialmente su estructura y composición, considerando que es el instrumento para la medida del sentido de la vida, desde claves logoterapéuticas, más utilizado hoy en día a efectos de investigación. Por otra parte, los resultados obtenidos pueden servir como punto de partida para llevar a cabo análisis de congruencia, de validez convergente y de validez discriminante del PIL-10, así como para establecer los baremos que permitan distinguir niveles de sentido de la vida, desde el vacío existencial al logro de sentido.

\section{Referencias}

Arribas, J. (2013). Hacia un modelo causal de las dimensiones del estrés académico en estudiantes de enfermería. Revista de Educación, 360. Publicación anticipada en línea. doi:10-4438/1988592X-RE-2011-360-126

Bentler, P. M. \& Bonnet, D. G. (1980). Significance tests and goodness-of-fit in the analysis of covariance structures. Psychological Bulletin, 88(3), 588-606. doi:10.1037//0033-2909.88.3.588

Bentler, P. M. (2006). EQS 6 Structural Equations Program Manual. Encino, CA: Multivariate Software, Inc.

Browne, M. W. \& Cudeck, R. (1993). Alternative ways of assessing model fit. En K. A. Bollen \& J. S. Long (Eds.), Testing structural equation models (pp. 136162). Newbury Parck, CA: Sage.

Conchado, A. (2011). Modelización multivariante de los procesos de enseñanza-aprendizaje basados en competencias de educación superior. Tesis Doctoral, Universidad Politécnica de Valencia, Valencia, España. Recuperado el 30 de marzo de 2012, de http:// riunet.upv.es/bitstream/handle/10251/12099/tesisUPV3654.pdf?sequence $=6$

Crumbaugh, J. C. (1968). Cross-validation of Purpose in Life Test based on Frankl's concepts. Journal of Individual Psychology, 24(1), 74-81.

Crumbaugh, J. C. \& Maholick, L. T. (1964). An experimental study in existentialism: The psychometric approach to Frankl's concept of noogenic neurosis. Journal of Clinical Psychology, 20(2), 200. 207. doi:10.1002/1097-4679(196404)20:2<200: AID-JCLP2270200203>3.0.CO;2-U

Crumbaugh, J. C. \& Maholick, L. T. (1969). Manual of instructions for the Purpose in Life Test. Saratoga, CA: Viktor Frankl Institute of Logotherapy.

Doerries, L. E. (1970). Purpose in life and social participation. Journal of Individual Psychology, 26(1), 50-53.

Dufton, B. C. \& Perlman, D. (1986). The association between religiosity and the Purpose-In-Life Test: Does it reflect purpose or satisfaction? Journal of Psychology and Theology, 14(1), 42-48.

Frankl, V. E. (1994). La voluntad de sentido. Barcelona: Herder. 
Frankl, V. E. (1997). Teoría y terapia de las neurosis. Barcelona: Herder.

Frankl, V. E. (2003). Ante el vacío existencial. Barcelona: Herder.

Gallego-Pérez, J. F. \& García-Alandete, J. (2004). Sentido en la vida y desesperanza en un grupo de estudiantes universitarios. Nous. Boletín de Logoterapia y Análisis Existencial, 8, 49-64.

García-Alandete, J., Gallego-Pérez, J. F. \& Pérez-Delgado, E. (2009). Sentido de la vida y desesperanza: un estudio empírico. Universitas Psychologica, 8(2), 447-454.

García-Alandete, J., Rosa, E., Soucase, B. \& GallegoPérez, J. F. (2011). Diferencias asociadas al sexo en las puntuaciones total y factoriales del Purpose-InLife Test en universitarios españoles. Universitas Psychologica, 10(3), 927-938.

Guibert, W. \& Del Cueto, E. R. (2003). Factores psicosociales de riesgo de la conducta suicida [Versión electrónica]. Revista Cubana de Medicina General Integral, 19(5). Recuperado el 25 de mayo de 2010, de http://bvs.sld.cu/revistas/mgi/vol19_5_03/ mgi04503.htm

Hair, J. F., Anderson, R. E., Tatham, R. L. \& Black, W. C. (2007). Análisis multivariante. Madrid: Prentice Hall.

Jackson, L. E. \& Coursey, R. D. (1988). The relationship of God control and internal locus of control to intrinsic religious motivation, coping and purpose in life. Journal for the Scientific Study of Religion, 27(3), 399-410. doi:10.2307/1387378

Jonsén, E., Fagerström, L, Lundman, B., Nygren, B., Vähäkangas, M. \& Strandberg, G. (2010). Psychometric properties of the Swedish version of the Purpose in Life Scale. Scandinavian Journal of Caring Sciences, 24(1), 41-48. doi:10.1111/j.14716712.2008.00682.x

Magaña, L., Zavala, M. A., Ibarra, I., Gómez, M. T. \& Gómez, M. M. (2004). El sentido de la vida en estudiantes de primer semestre de la Universidad de La Salle Bajío. Revista del Centro de Investigación, 6(22), 5-13.

Marsh, A., Smith, L., Piek, J. \& Saunders, B. (2003). The Purpose In Life Scale: Psychometric properties for social drinkers and drinkers in alcohol treatment.
Educational EF Psychological Measurement, 63(5), 859-871. doi: $10.1177 / 0013164402251040$

McGregor, I. \& Little, B. R. (1998). Personal projects, happiness, and meaning: On doing well and being yourself. Journal of Personality and Social Psychology, 74(2), 494-512. doi:10.1037//0022-3514.74.2.494

Meier, A. \& Edwards, H. (1974). Purpose-InLife Test: Age and sex differences. Journal of Clinical Psychology, 30(3), 384-386. doi:10.1002/1097-4679(197407)30:3<384::AIDJCLP2270300351>3.0.CO;2-V

Melton, A. M. A. \& Schulenberg, S. E. (2007). On the relationship between meaning in life and boredom proneness: Examining a logotherapy postulate. Psychological Reports, 101(3F), 1016-1022. doi:10.2466/pro.101.4.1016-1022

Melton, A. M. A. \& Schulenberg, S. E. (2008). On the measurement of meaning: Logotherapy's empirical contributions to Humanistic psychology. The Humanistic Psychologist, 36(1), 31-44. doi:10.1080/08873260701828870

Molasso, W. R. (2006). Exploring Frankl's Purpose in Life with college students. Journal of College and Character, 7(1), 1-10. doi:10.2202/1940-1639.1502

Molcar, C. C. \& Stuempfig, D. W. (1988). Effects of world view on purpose in life. The Journal of Psychology, 122(4), 365-371. doi:10.1080/00223980.1 988.9915523

Morgan, J. E. \& Farsides, T. L. (2009). Measuring meaning in life. Journal of Happiness Studies, 10(2), 197214. doi:10.1007/s10902-007-9075-0

Noblejas de la Flor, M. A. (1994). Logoterapia. Fundamentos, principios y aplicación. Una experiencia de evaluación del "logro interior de sentido". Tesis Doctoral, Universidad Complutense de Madrid, Madrid, España. Recuperado el 13 de mayo de 2007, de http://eprints.ucm.es/tesis/19911996/S/5/ S5005701.pdf

Noblejas de la Flor, M. A. (2000). Fiabilidad de los tests PIL y Logotest. Nous. Boletín de Logoterapia y Análisis Existencial, 4, 81-90.

Nunnally, J. C. \& Bernstein, I. (1994). Psychometric theory. New York NY: McGraw-Hill.

Nygren, B., Alex, L., Jonsén, E., Gustavsson, Y., Norberg A. \& Lundman B. (2005). Resilience, sense of coherence, purpose in life and self-transcendence in 
relation to perceived physical and mental health among the oldest old. Aging $\mathcal{E}$ Mental Health, 9(4), 354-362. doi:10.1080/1360500114415

Preble, J. (1986). The LOGO-Test: First North American norms. En F. H. Jones \& J. Jones (Eds.), Proceedings of the fifth world congress of logotherapy (pp. 196-217). Berkeley, CA: Institute of Logotherapy Press.

Reker, G. T. (2000). Theoretical perspectives, dimensions, and measurement of existential meaning. En G. T. Reker \& K. Chamberlain (Eds.), Exploring existential meaning: Optimizing human development across the life span (pp. 39-55). Thousand Oaks, CA: Sage.

Reker, G. T. \& Chamberlain, K. (2000). Existential Mediating: Reflections and directions. En G. T. Reker \& K. Chamberlain (Eds.), Exploring existential meaning: Optimizing human development across the life span (pp. 199-209). Thousand Oaks, CA: Sage.

Rennie, K. M. (1997, enero). Exploratory and confirmatory rotation strategies in exploratory factor analysis. Comunicación presentada al Encuentro Anual de la Southwest Educational Research Association, Austin, Texas, USA. Recuperado el 31 de mayo de 2009, de http://mirror.eschina.bnu.edu.cn/Mirror1/ accesseric/ericae.net/ft/tamu/Rota.htm

Risco, A. (2009). Sentido de la vida: evolución e implicaciones para la Educación Superior. Tesis Doctoral inédita, Universidad de Salamanca, Salamanca, España.

Robbins, M. \& Francis, L. J. (2005). Purpose in life and prayer among Catholic and Protestant adolescents in Northern Ireland. Journal of Research on Christian Education, 14(1), 73-93. doi:10.1080/10656210509484981

Sallee, D. T. \& Casciani, J. M. (1976). Relationship between sex drive and sexual frustration and purpose in life. Journal of Clinical Psychology, 32(2), 273-275. doi:10.1002/1097-4679(197604)32:2<273::AIDJCLP2270320214>3.0.CO;2-S

Schulenberg, S. E. (2004). A psychometric investigation of logotherapy measures and the outcome questionnaire (OQ-45.2). North American Journal of Psychology, 6(3), 477-492.

Schulenberg, S. E. \& Melton, A. M. A. (2010). A confirmatory factor-analytic evaluation of the PurposeIn-Life Test: Preliminary psychometric support for a replicable two-factor model. Journal of Happiness Studies, 11(1), 95-111. doi:10.1007/s10902-0089124-3

Shek, D. T. L. (1988). Reliability and factorial structure of the Chinese version of the Purpose-In-Life Questionnaire. Journal of Clinical Psychology, 44(3), 384-392. doi:10.1002/1097-4679(198805)44:3<384::AIDJCLP2270440312>3.0.CO;2-1

Steger, M. F. (2006). An illustration of issues in factor extraction and identification of dimensionality in psychological assessment data. Journal of Personality Assessment, 86(3), 263-272. doi:10.1207/ s15327752jpa8603_03

Waisberg, J. L. \& Starr, M. W. (1999). Psychometric properties of the Purpose-In-Life Test with a sample of substance abusers. International Forum for Logotherapy, 22(1), 22-26.

Wald, A. (1943). Tests of statistical hypotheses concerning several parameters when the number of observations is large. Transactions of the American Mathematical Society, 54(3), 426-482. doi:10.1090/ S0002-9947-1943-0012401-3

Walters, L. H. \& Klein, A. E. (1980). A cross-validated investigation of the Crumbaugh Purpose-In-Life Test. Educational and Psychological Measurement, 40(4), 1065-1071. doi:10.1177/001316448004000434 


\title{
Apéndice
}

\author{
Purpose-In-Life Test (Parte A)
}

Crumbaugh y Maholick (1969) versión de Noblejas de la Flor (1994)

Se muestra el enunciado de los ítems y los anclajes de los puntos 1 y 7 (el anclaje

del punto 4 es Neutral y el resto de puntos carecen de anclaje).

1. Generalmente me encuentro: Completamente aburrido / Exuberante, entusiasmado.

2. La vida me parece: Completamente rutinaria / Siempre emocionante.

3. En la vida tengo: Ninguna meta o anhelo / Muchas metas y anhelos definidos.

4. Mi existencia personal es: Sin sentido ni propósito / Llena de sentidos y propósitos.

5. Cada día es: Exactamente igual / Siempre nuevo y diferente.

6. Si pudiera elegir: Nunca habría nacido / Tendría otras nueve vidas iguales a esta.

7. Después de retirarme: Holgazanearía el resto de mi vida / Haría las cosas emocionantes que siempre deseé realizar.

8. En el logro de mis metas vitales: No he conseguido ningún progreso / He llegado a mi realización completa.

9. Mi vida es: Vacía y llena de desesperación / Un conjunto de cosas buenas y emocionantes.

10. Si muriera hoy, me parecería que mi vida ha sido: Una completa basura / Muy valiosa.

11. Al pensar en mi propia vida: Me pregunto a menudo la razón por la que existo / Siempre encuentro razones para vivir.

12. Tal y como yo lo veo en relación con mi vida, el mundo: Me confunde por completo / Se adapta significativamente a mi vida.
13. Me considero: Una persona irresponsable / Una persona muy responsable.

14. Con respecto a la libertad de que dispone para hacer sus propias elecciones, creo que el hombre es: Completamente esclavo de las limitaciones de la herencia y del ambiente / Absolutamente libre de hacer todas sus elecciones vitales.

15. Con respecto a la muerte, estoy: Falto de preparación y atemorizado / Preparado y sin temor.

16. Con respecto al suicidio: Lo he considerado seriamente como una salida a mi situación / Nunca le he dedicado un segundo pensamiento.

17. Considero que mi capacidad para encontrar un significado, un propósito o una misión en la vida es: Prácticamente nula / Muy grande.

18. Mi vida está: Fuera de mis manos y controlada por factores externos / En mis manos y bajo mi control.

19. Enfrentarme a mis tareas cotidianas supone: Una experiencia dolorosa y aburrida / Una fuente de placer y satisfacción.

20. He descubierto: Ninguna misión o propósito en mi vida / Metas claras y un propósito satisfactorio para mi vida. 